Monatsschrift f. Geburtshülfe u. Gynäkologie 1915;42:284-285

\title{
Buchanzeige
}

Max Hirsch-Berlin: Fruchtabtreibung und Präventivverkehr im Zusammen-hang mit dem Geburtenrückgang. Würzburg 1914. Curt Kabitzsch.

In vorliegende $\Gamma$ Arbeit hat Verf. die zahlreichen kleineren Arbeiten zusammengefaßt, die er über diesen Gegenstand verfertigt hat. Die offiziellen Statistiken geben kein rechtes Bild von der tatsächlichen Aus-breitung der Fruchtabtreibung. Mehr als $80 \mathrm{pCt}$. der Aborte sind der kriminellen Entstehung zum mindesten verdächtig. Es handelt sich hierbei um die Vernichtung von Kindern, sowie um die Entstehung von chronischen Unterleibsleiden und Sterilität. Verf. behauptet, daß neben den Geschlechts-krankheiten die Fruchtabtreibung mit ihren Folgen die größte Bedeutung in der Atiologie der chronischen Unterleibsentzündungen und der Sterilität zukommt. Die Motive der Fruchtabtreibung sind verschieden. Bei unehe-lich Geschw $1 / 8$ ngerten der besseren Stände sind es Scham, Furcht vor Schande und Verlust der bürgerlichen Stellung, im Arbeiterstand pflegen meist wirtschaftliche Motive maßgebend zu sein. Der

Geburtenrückgang scheint jedoch durch die Abtreibungen der unehelich Geschwängerten nicht hervor-gerufen zu sein, da die Zahl der unehelichen Kinder dauernd zunimmt. Der größte Teil der Abtreibungen wird in der Ehe vorgenommen. Es scheint, daß die Mittel zur Beschränkung der Kinderzahl in der Ehe in den oberen Ständen der Präventivverkehr, in den unteren Klassen die Vernichtung der Frucht sind, und daß der Geburtenrückgang hierin seine Ursachen hat. Innerhalb der Ehe entspringt die Veranlassung zur Fruchtabtreibung meist einem wirtschaftlichen Notstand. Wesentlich ist hierbei das Anwachsen der Beteiligung der Frau am Erwerbsleben. Von Bedeutung sind auch die Bestimmungen der Behörden über das Heiraten der Beamten (Offiziere) und Beamtinnen, ferner die zahlreichen Ehescheidungen. Hinzu kommt die Furcht vor der Entbindung - speziell nach schweren Ent-bindungen, die Schädigung des Organismus durch das gewerbliche Leben (Chlorose, Beckenanomalien), die Furcht vor kranker Nachkommenschaft. Der 2. Teil der Arbeit handelt vom Geburtenrückgang. Dieser ist in fast alien europäischen Ländern zu verzeichnen. Verf. gibt hierüber genaue statistische Angaben. Es kommen im allgemeinen nur die ehelichen G $\theta$-burten in Betracht. Der Geburtenrückgang ist in den Städten besonders stark, auf dem Lande hat er erst später eingesetzt. Im 3. Teil werden die Mittel besprochen, die im Kampf gegen die Fruchtabtreibung und den Geburtenrückgang anzuwenden sind. Ein Verbot der antikonzeptionellen Mittel ist unratsam, ebenso das Aufheben der Schweigepflicht des Arztes. Im Vordergrund steht der weitere Ausbau der sozialen Hygiene und die großzügige Pflege einer rationellen Fortpflanzungs- und Rassenhygiene. Auf diesem ümwege ist eine Aufbesserung der Fruchtbarkeit und Geburten-häufigkeit zu erwarten. Das bedeutendste Mittel im Kampfe gegen die Fruchtabtreibung ist die generative Prophylaxe: Schwangerschaftsverbot und Schwangerschaftsverhütung aus therapeutischen und prophylaktischen Gründen. Verf. will auch aus prophylaktischen Gründen zum Beispiel die Schwangerschaft unterbrochen wissen. Er führt hiermit etwas Neues in die medizinische Wissenschaft ein, vor dem man sich mit Recht bis jetzt gescheut hat. Rassehygienische Indikationen zur Einleitung des Abortes anzuerkennen, wozu der Verf. rät, müssen wir entschieden zurückweisen. 


\section{Literatur-Verzeichni $\beta$.}

285

Hierdurch würde m. E. gerade das Gegenteil von dem erreicht, was Verf. beabsiehtigt. Die Lehre von der Eugenik, für die Verf. eintritt, ist so dehnbar und kann soviel Verwirrung anrichten, daß sie bei dem ersichtlichen Be-steeben der Menschen, die Kinderzahl herabzusetzen, materiell nuГ zu einer Verntehrung der Abtreibung dienen wird.

Die ungemein genaue Arbeit wird sicher zu einer lebhaften Diskussion

führen. Wer sie liest, gewinnt einen guten Überblick über die Frage des

Geburtenrückganges. Hantke- Berlin.

Literatur-Verzeichnis.

Geburtshülfe.

Robert von Olshausens wissenschaftliches Lebenswerk, dargestellt von seinem Schüler G.

Winter. Stuttgart 1915. Union: Deutsche Verlags-gesellschaft.

Eugen Kòberlé. Nachruf von J. Klein. Zbl. f. Gyn. No. 32.

Böhi. Über Chorioma malignum. Arch. f. Gyn. 104. S. 214.

Döderleín, A. Handbuch der Geburtshülfe. Bearbeitet von K. Baisch,

A. Döderlein, M. Hofmeier, Ph. Jung, J. Ibrahim, R. Kockel, B. Krönig,

0. Küstner, C. Menge, M. Neu, M. v. Pfaundler, O. Sarwey, L. Seitz,

H. Sellheim, E. Siemerling, F. Graf Spee, J. Veit, F. Weber, W.

Zangemeister, P. Zweifel in 3 Bänden, herausgegeben $3 / 8$ von A. Döderlein.

Wiesbaden. 1915. J. F. Bergmann.

Erster Band. Anatomie und Physiologie der Schwangerschaft von

Graf Spee, O. Sarwey; Diagnose der Schwangerschaft von M. Neu. -

Physiologie der Geburt von H. Sellheim. - · Physiologie des Wochen-

bettes von Ph. Jung. - - Physiologie des Neugeborenen von M. von

Pfaundler. 257 - Abbildungen im Text und 4 Tafeln. Freund, Herm. Geburt nach konservativer

Ovariotomie. Hiluscyste des

Eierstocks. Zbl. f. Gyn. 24. VII. S. 523. Jaschke, R. Th. Akute puerperale Uterusinversion. Zbl. f. Gyn. 7. VIII.

S. 557. Küstner, O. Extraperitonealer Kaiserschnitt wegen verschleppter Quer-

lage. Zbl. f. Gyn. 31. VII. S. 539. Lanz. Über einen Fall von Tuberkulose der Placenta und der Eihäute. Arch.

f. Gyn. 104. S. 238. Wamekros. Zur Prognose der puerperalen Fiebersteigerungen a\%oif Grund bakteriologischer und histologischer Untersuchungen. Arch. f. Gyn.

104. S. 301. Werner. Über einen seltenen Fall von Zwergwuchs. Arch. f. Gyn. 104.

S. 278.

Gynäkologie. Burrows, W. F. Kontraindikationen gegen die intraabdominale Anwendung

von Öl. Med. Rec. 10. VII. S. 66. Edmonds, Agnes M. Eine uniloculare Ovarialcyste. Journ. Am. med. Assoc.

10. VII. S. 164. Fraenkel, L. Ätiologie und Therapie von Frauenkrankheiten bei Irren.

Med. Klin. 18. VII. S. 799 und 25. VII. S. 828. 